Editorial

\title{
When your heart depends on your gut feelings
}

\section{Cuando el corazón depende del tracto digestivo}

\author{
Lucia Musumeci, ${ }^{a}$ Cécile Oury, ${ }^{a}$ and Patrizio Lancellotti ${ }^{\mathrm{a}, \mathrm{b}, *}$ \\ a Department of Cardiology and Cardiovascular Surgery, University of Liège Hospital, GIGA Cardiovascular Sciences, CHU Sart Tilman, Liège, Belgium \\ ${ }^{\mathrm{b}}$ Gruppo Villa Maria Care and Research, Maria Cecilia Hospital, Cotignola, and Anthea Hospital, Bari, Italy
}

The epidemiology, microbiology, and diagnosis of infective endocarditis (IE) has changed rapidly over the last 50 years. The International Collaboration on Endocarditis (ICE) study ${ }^{1}$ reported that the most prevalent causes of IE were degenerative valve disease and the presence of prosthetic valves (in the 1960s and 1970s the main causes were rheumatic lesions). Moreover, the very recent EURO-ENDO observational study has highlighted a change in the microbiology of IE-the most prevalent causative microorganisms are staphylococci (44\%), followed by enterococci (15.8\%), oral streptococci (12.3\%), and Streptococcus gallolyticus (6.6\%)-and enterococci are no longer the third cause of IE, as they were at the time of the ICE study, and have become the second cause. This is probably due to the increasing proportion of older patients with comorbidities affected by IE, which also makes IE a disease with poor prognosis. ${ }^{2}$

Gut permeability and inflammation, being more prevalent in the older population, could explain gut bacteria "leakage" in the systemic circulation, potentially causing not only cardiovascular but also autoimmune diseases. ${ }^{3}$ Enterococci are the most abundant bacteria in the human gastrointestinal tract (small and large intestine), representing $1 \%$ of the human fecal flora, with Enterococcus faecalis and Enterococcus faecium being the ${ }^{2}$ main species of the enterococcus genus. In healthy individuals, enterococci play a commensal/probiotic role but, in elderly people, who are more frequently affected by dysbiosis (an imbalance in the community of healthy human gut microbiota), bacteria can cross the mucosa of the gut and enter the circulation. One of the many ways the microbiota maintain intestinal homeostasis is by stimulating goblet cell secretion of mucin, which is a thick layer protecting the colonic epithelium from intestinal microbes. Whenever this commensal role is not achieved, bacteria can enter the circulation and colonize other areas of the body and form biofilms. ${ }^{4}$ Chronic dysbiosis associated with other factors, such as genetics and diet-derived chemical irritants, can induce chronic colonic inflammation and lead to colorectal diseases and colorectal neoplasia (CRN), which can progress to colorectal cancer (CRC). ${ }^{5}$

Enterococci are not the only bacteria able to cross the mucosa of the colon, S. gallolyticus, which is, however, not present in most human gut (2.5\%-15\% of healthy humans), also has this property. S. gallolyticus bacteremia has been extensively studied and the mechanism of translocation and dissemination is well understood. Translocation happens through surface-exposed adhesins allowing

SEE RELATED CONTENT:

https://doi.org/10.1016/j.rec.2019.07.007

* Corresponding author: Department of Cardiology, University of Liège Hospital, Domaine Universitaire du Sart Tilman, B35, 4000 Liège, Belgium.

E-mail address: plancellotti@chuliege.be (P. Lancellotti). adhesion to host cells, whereas dissemination involves the pilus (Pil3 expression), which binds to plasma fibrinogen. Once in the blood stream, Pil1 expression is responsible for the binding to collagen I (present on the surfaces of damaged heart valves), achieving heart colonization. ${ }^{6}$ As long ago as the 1970 s, S. gallolyticus bacteremia was found to be associated with both CRN and IE. ${ }^{7}$

In contrast, the mechanism of E. faecalis bacteremia is less well known. Although the main portal of entry of E. faecalis is the genitourinary tract, it has been shown that E. faecalis overgrowth can stimulate reactive oxygen species production and induce genomic instability in colonic cells, favoring lesions of the intestinal mucosa and translocation of $E$. faecalis in the blood circulation $^{8}$ (figure 1 ).

Supporting the hypothesis of the gastroenteric tract as a portal of entry of E. faecalis bacteremia, a study published in 2017 by Pericàs et al. explored the relationship between E. faecalis IE (EFIE) and CRN. These authors examined the prevalence of colorectal neoplasms in EFIE patients whose presumed source of infection could not be determined and found that $50.8 \%$ had CRN. In the group of patients with an identified portal of entry of the infection, only 6 patients underwent colonoscopy and 1 of them was diagnosed with CRN (16.7\%); therefore no conclusions could be drawn for this group. ${ }^{9}$

In an article by Escolà-Vergé et al., ${ }^{10}$ recently published in Revista Española de Cardiología, the authors aimed to determine the prevalence of colorectal disease in EFIE patients, irrespective of the hypothesized source of infection. The rationale behind their study was based on the fact that $5 \%$ of patients with IE (all etiologies) would experience recurrent infection with a higher mortality risk, the reason being a potential undiagnosed colorectal disease (portal of entry of bacteria into the circulation). If this hypothesis is correct, performing a colonoscopy, which could identify and treat the upstream cause of the endocarditis, would decrease the risk of recurrence. In their study, the authors included all consecutive episodes $(n=103)$ with EFIE seen in 4 referral centers ( 3 centers in Spain and 1 in Italy). Patients were classified based on the presumed source of infection into patients with "unknown portal of entry" and patients with "known portal of entry". Only 70 EFIE patients underwent colonoscopy (6 months before or after the EFIE diagnosis): patients with an "unknown portal of entry" were more numerous than those with an "known portal of entry" (64\% vs 36\%).

Comparison of demographic and clinical characteristics between the 2 groups revealed that health care-associated infections were more prevalent in patients with a "known portal of entry" than in those with an "unknown portal of entry" (83\% vs $29 \%$, 